\title{
Information: Subjective or Objective? ${ }^{\dagger}$
}

\author{
Ron Cottam ${ }^{1, *}$, Willy Ranson ${ }^{2}$ and Roger Vounckx ${ }^{1}$ \\ 1 The Living Systems Project, Department of Electronics and Informatics, Vrije Universiteit Brussel (VUB), \\ Pleinlaan 2, 1050 Brussels, Belgium; rvounckx@etrovub.be \\ 2 IMEC vzw, Kapeldreef 75, 3001 Leuven, Belgium; wranson@etrovub.be \\ * Correspondence: ricottam@etrovub.be; Tel.: +32-2-629-2933 \\ + Presented at the IS4SI 2017 Summit DIGITALISATION FOR A SUSTAINABLE SOCIETY, Gothenburg, \\ Sweden, 12-16 June 2017. \\ Published: 8 June 2017
}

\begin{abstract}
We consider the derivation of information in both organic and inorganic systems, that is both for organisms and solid state physics, and conclude that information is always subjective, although this presents less of a problem in inorganic systems, where the simplicity of a measurement's ecosystem implies that information tends towards being objective.
\end{abstract}

Keywords: information; hierarchy; hyperscale; metascale; solid state physics

\section{Introduction}

The target of this paper is to draw a comparison between information in living systems and information in inorganic contexts. The two representative contexts we will refer to are those of an organism and of solid state physics. We have published extensively elsewhere on the properties of a model hierarchy (e.g., [1]; and most recently [2,3]), and to reproduce the entire derivation would be inappropriate for this extended abstract. Consequently, we will restrict ourselves to the brief sketch which follows, in the hope that this attenuated version is sufficiently comprehensible.

\section{Natural Model Hierarchy}

Figure 1 illustrates the general form of a model hierarchy in the example of an organism. The hierarchy is pictured 'on its side' to support the view that no one level of organization is ultimately dominant. Each organizational level constitutes a model of an entire organism at a specific structural scale, as indicated, and as opposed to conventional hierarchical representation, for example Salthe's [4] compositional hierarchy, where a single constitutional element is presented at each level. The model hierarchy appears to be the parent [5] of both compositional hierarchy and subsumption hierarchy [4]. Although Figure 1 is presented in structural terms, such a hierarchy can also be represented in terms of function, and it automatically includes any required aspects of heterarchy. Regions between adjacent levels are extremely complex in Rosen's [6] sense, and these embody the locally scaled internal representations of the organism's ecosystem. Each organizational level is partially closed and partially open to the rest of the hierarchy, and direct access to individual levels from outside or from other internal levels is limited.

The hierarchy automatically creates a pair of approximate models of itself [5], one for the organizational levels, one for the complex regions (see Figure 2), which we refer to as hyperscales [7].

Ultimately these integrate, giving a singular metascalar representation (as illustrated in Figure 3) which is the real identity of the organism [5]. This corresponds with the identification of information with the integration of data (the model level hyperscale) and context (the complex region hyperscale) [2]. 


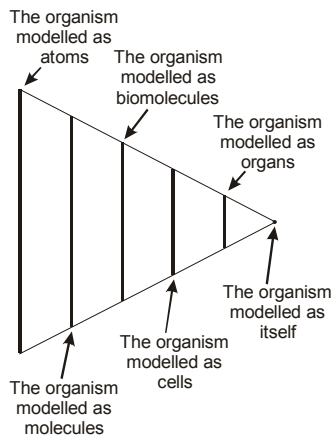

Figure 1. The general form of a model hierarchy as imagined for an organism.

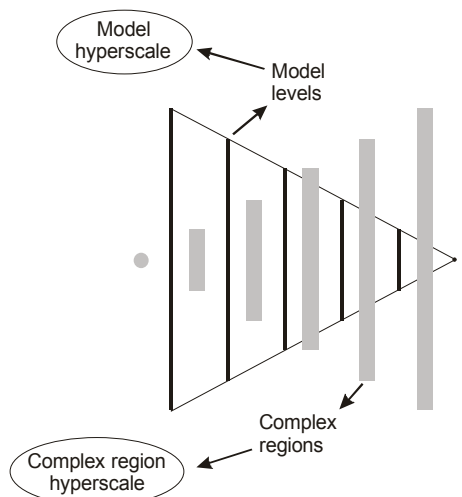

Figure 2. The generation of approximate hyperscalar models of the two partial hierarchies.

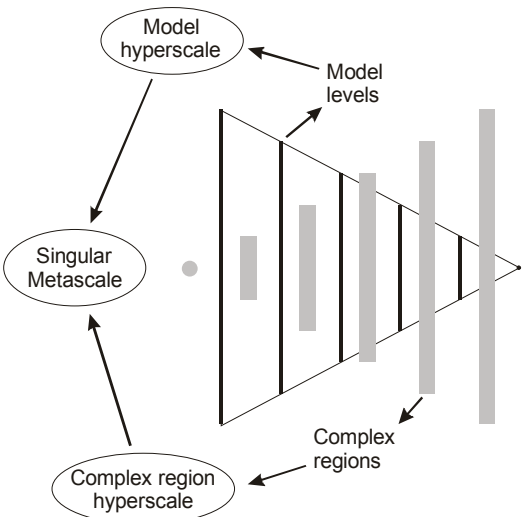

Figure 3. The two hyperscales integrate to a singular metascale, which is the real nature of the organism.

\section{Solid State Physics}

Our next task is to describe formulation of the electronic properties of solid state physics. We start with a single atom, which exhibits a sequence of electron energetic levels spreading outward from the nucleus, as indicated in Figure 4.

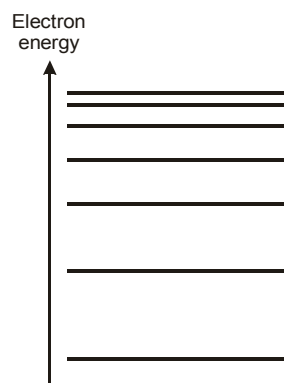

Figure 4. Electron energy levels around the atomic nucleus. 
In a crystal consisting of a myriad of closely packed atoms these energy levels broaden, following the Pauli exclusion principle [8], to a set of energetic bands, as illustrated in Figure 5. Adjacent bands are separated by 'forbidden regions' as indicated.

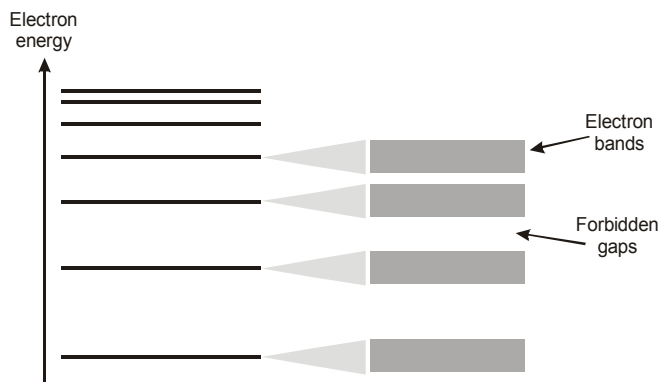

Figure 5. Spreading of the atomic electron energy levels into bands separated by 'forbidden regions'.

A parallel mathematical derivation of this context following the Kronig-Penney model [8] yields the equivalent arrangement of bands shown in Figure 6.

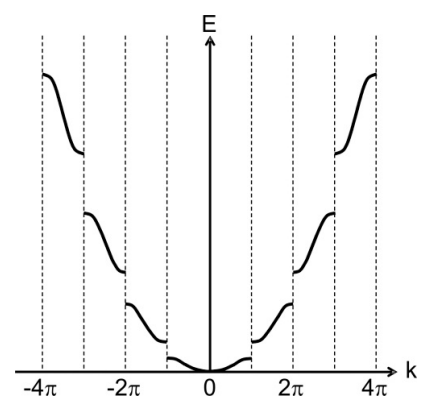

Figure 6. The electronic band structure derived from the Kronig-Penney model. E is electron energy;

$\mathrm{k}$ is electron momentum.

Similarity between the electronic band structure and the organism model hierarchy is already apparent. The horizontally extended regions of Figure 6-called Brillouin zones-fold in to the central region to give Figure 7 -an apparent equivalence to the generation of the model-level hyperscale in an organism.

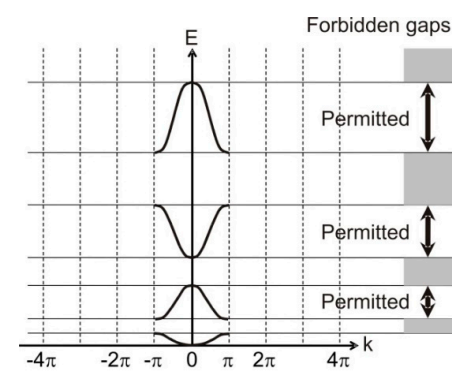

Figure 7. Folding-in of the Brillouin zones into the central region.

The second ecosystemic hyperscale is here relatively simple, as in the inorganic case it collapses down to a simple set of parameters of temperature, pressure, magnetic field, ... At the highest level of organization, electronic conduction is controlled by two aspects. Firstly, the electrons themselves, secondly, the regions from which they may be absent, called 'holes'. The holes' properties are those of the electrons' ecosystems. Consequently, conductivity in a crystal is derived from the properties of both electrons and their ecosystems - an apparent mirroring of metascale in an organism. 


\section{Comparing Natural Hierarchy and Solid State Physics}

The coupling feature between these two systems appears to be the relationship between process and structure, where both are intimately connected in an organism, and where process is virtually absent from a crystal. This gives us a way of characterizing system properties, including information, in the two situations. We believe that the higher level characteristic of complementarity can best represent this coupling, and that objectivity or subjectivity in a given context can be referred to the degree of its presence in a context, or of its absence.

Figure 8 attempts to picture this relationship. It should be noted that we do not suggest that the implied coupling is linear: this is shown in the Figure merely to establish the sense of the relationship between complementarity and objectivity/subjectivity. Interestingly, this relationship resembles one that we have published earlier [5] between complementarity and the excluded or exclusive middle in bilateral contexts, such as in Brenner's [9] Logic in Reality (see Figure 9). Increase in system complementarity corresponds to a decrease in the accessibility of the bilateral 'end-points' of the system, and a focusing of system properties on the mid-region.

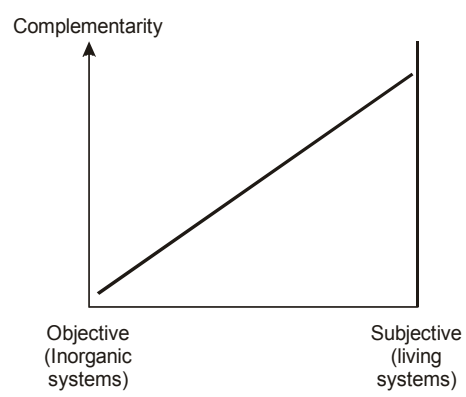

Figure 8. A suggested relationship between complementarity and subjectivity and objectivity, linked to organisms and inorganic systems.

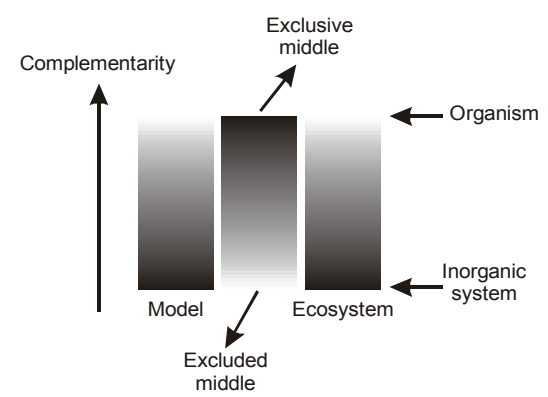

Figure 9. Change from the excluded middle to the exclusive middle with increase in system complementarity.

We have earlier published an investigation of information in living systems (Cottam et al., 2016) which concluded, following Havel's [10] propositions of the natures of subjectivity and objectivity, that information in organisms is fundamentally subjective, and exclusive in character. This corresponds to the preponderance of complementarity in living systems, as we indicated in Figure 8. On the other hand, complementarity in inorganic systems is lacking, and measures of information derived in these systems are fundamentally objective, in Havel's [10] sense.

Conflicts of Interest: The authors declare no conflict of interest.

\section{References}

1. Cottam, R.; Ranson, W.; Vounckx, R. Autocreative hierarchy II: Dynamics-Self-organization, emergence and level-changing. In Proceedings of the International Conference on Integration of Knowledge Intensive Multi-Agent Systems, Cambridge, MA, USA, 30 September-4 October 2003; Hexmoor, H., Ed.; IEEE: Piscataway, NJ, USA, 2003; pp. 766-773.

2. Cottam, R.; Ranson, W.; Vounckx, R. Hierarchy and the nature of information. Information 2016, 7, 1-10. 
3. Cottam, R.; Ranson, W.; Vounckx, R. Scaled ecosystemic Rosennean complexity. Ecol. Complex. 2017, doi:10.1016/j.ecocom.2017.02.001.

4. Salthe, S.N. Hierarchical structures. Axiomathes 2012, 22, 355-383.

5. Cottam, R.; Ranson, W.; Vounckx, R. A framework for computing like Nature. In Computing Nature; Springer SAPERE Series; Dodig-Crnkovic, G., Giovagnoli, R., Eds.; Springer: Heidelberg, Germany, 2013; pp. 23-60.

6. Rosen, R. Life Itself: A Comprehensive Inquiry into the Nature, Origin and Fabrication of Life; Columbia University Press: New York, NY, USA, 1991.

7. Cottam, R.; Ranson, W.; Vounckx, R. Life and simple systems. Syst. Res. Behav. Sci. 2005, 22, 413-430.

8. Azàroff, L.V.; Brophy, J.J. Electronic Processes in Materials; McGraw Hill: Tokyo, Japan, 1963.

9. Brenner, J.E. Logic in Reality; Springer: Berlin, Germany, 2008.

10. Havel, I.M. Scale dimensions in Nature. Int. J. Gen. Syst. 1996, 24, 295-324.

(C) 2017 by the authors. Licensee MDPI, Basel, Switzerland. This article is an open access article distributed under the terms and conditions of the Creative Commons Attribution (CC-BY) license (http://creativecommons.org/licenses/by/4.0/). 